\title{
Search for Galactic runaway stars using Gaia Data Release 1 and HIPPARCOS proper motions (Corrigendum)
}

\author{
J. Maíz Apellániz ${ }^{1}$, M. Pantaleoni González ${ }^{1,2}$, R. H. Barbá ${ }^{3}$, S. Simón-Díaz ${ }^{4,5}$, I. Negueruela ${ }^{6}$, \\ D. J. Lennon ${ }^{7}$, A. Sota ${ }^{8}$, and E. Trigueros Páez ${ }^{1,6}$ \\ ${ }^{1}$ Centro de Astrobiología CSIC-INTA, Campus ESAC, Camino bajo del castillo s/n, 28692 Villanueva de la Cañada, Spain \\ e-mail: jmaiz@cab.inta-csic.es \\ 2 Departamento de Astrofísica y Física de la Atmósfera, Universidad Complutense de Madrid, 28040 Madrid, Spain \\ 3 Departamento de Física y Astronomía, Universidad de La Serena, Av. Cisternas 1200 Norte, La Serena, Chile \\ 4 Instituto de Astrofísica de Canarias, 38200 La Laguna, Tenerife, Spain \\ 5 Departamento de Astrofísica, Universidad de La Laguna, 38205 La Laguna, Tenerife, Spain \\ 6 Departamento de Física, Ingeniería de Sistemas y Teoría de la Señal, Escuela Politécnica Superior, Universidad de Alicante, \\ Carretera San Vicente del Raspeig s/n, 03690 San Vicente del Raspeig, Spain \\ 7 ESA, European Space Astronomy Centre, Camino bajo del castillo s/n, 28692 Villanueva de la Cañada, Spain \\ 8 Instituto de Astrofísica de Andalucía-CSIC, Glorieta de la Astronomía s/n, 18008 Granada, Spain
}

A\&A, 616, A149 (2018), https://doi .org/10.1051/0004-6361/201832787

Key words. surveys - proper motions - Galaxy: structure - supergiants - stars: kinematics and dynamics - errata, addenda

The wrong versions of Figs. 1 and 2 were published in the paper. The points corresponding to Table 6 in the top panels were missing and a single "dummy" unlabeled point was shown instead. The correct versions are given here. 

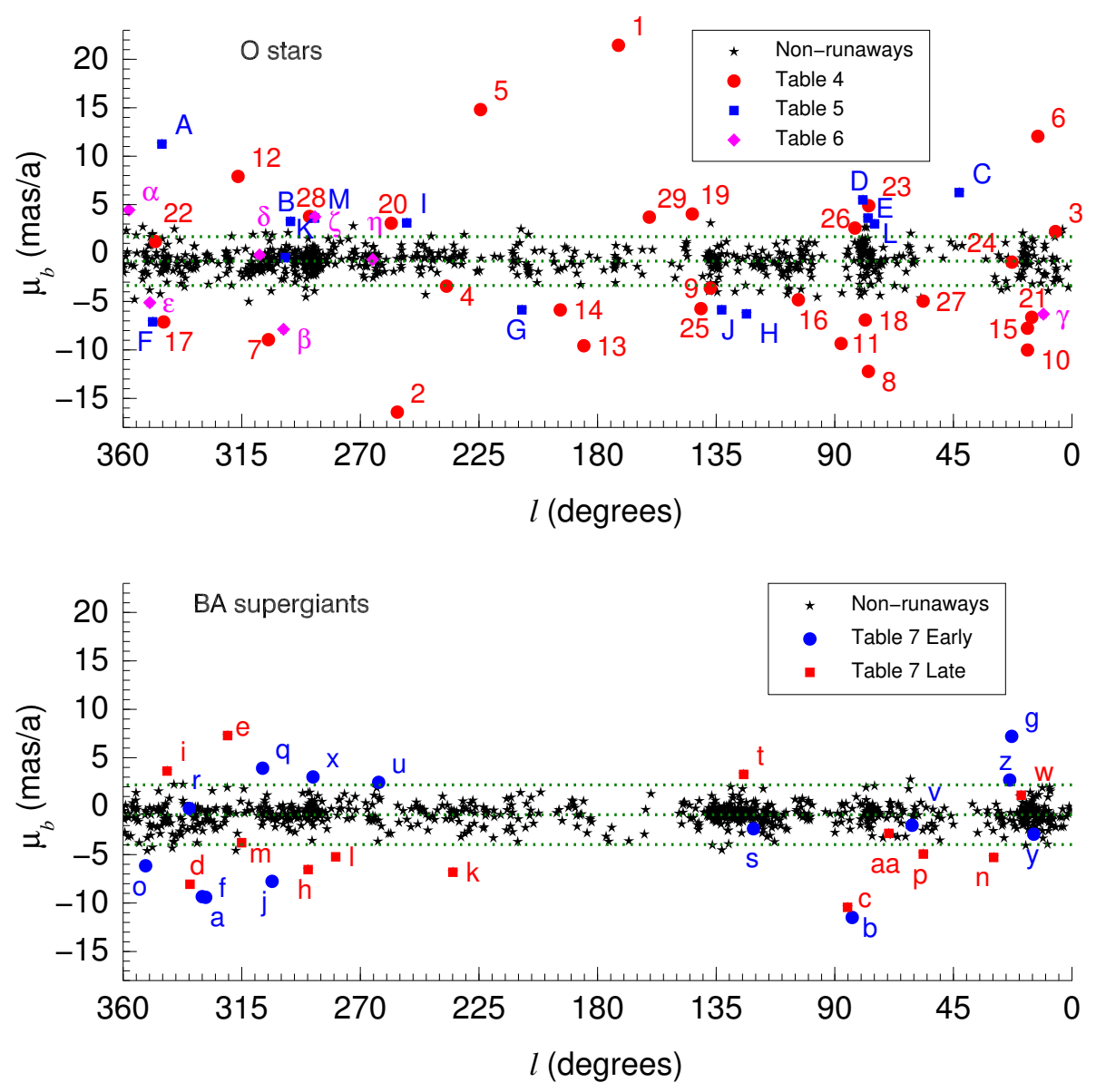

Fig. 1. Observed proper motion in Galactic latitude for $\mathrm{O}$ stars (top panel) and BA supergiants (bottom panel). Different colors and symbols are used to identify the runaway candidates in Tables 4-7, and the IDs in these tables are also shown. The dotted green lines represent the functions and $2 \sigma$ deviations used to detect runaway candidates. The vertical scales are the same in both panels to facilitate comparison.
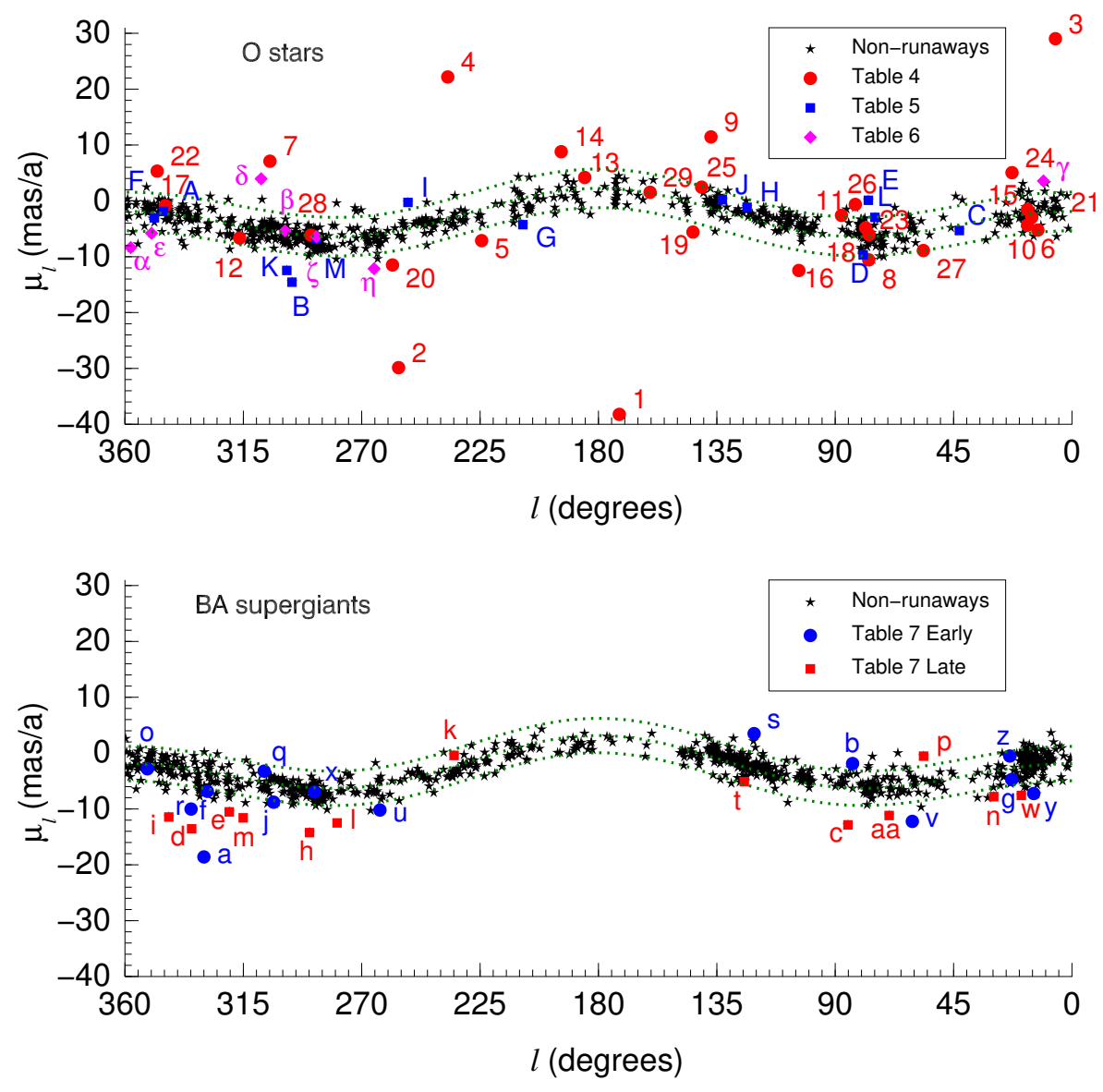

Fig. 2. Observed proper motion in Galactic longitude for $\mathrm{O}$ stars (top panel) and BA supergiants (bottom panel). Different colors and symbols are used to identify the runaway candidates in Tables 4-7, and the IDs in these tables are also shown. The dotted green lines represent the functions and $2 \sigma$ deviations used to detect runaway candidates. The vertical scales are the same in both panels to facilitate comparison. 